\title{
A phase II JN-I-10 efficacy study of IDRF- based surgical decisions and stepwise treatment intensification for patients with intermediate-risk neuroblastoma: a study protocol
}

Tomoko lehara ${ }^{1 *}$, Akihiro Yoneda ${ }^{2}$, Atsushi Kikuta ${ }^{3}$, Toshihiro Muraji ${ }^{4}$, Kazuaki Tokiwa ${ }^{5}$, Hideto Takahashi ${ }^{6}$, Satoshi Teramukai', Tetsuya Takimoto ${ }^{8}$, Shigeki Yagyu' ${ }^{1}$, Hajime Hosoi ${ }^{1}$, Tatsuro Tajiri ${ }^{5}$ and the Japan Children's Cancer Group Neuroblastoma Committee

\begin{abstract}
Background: Few clinical trials have been reported for patients with intermediate-risk neuroblastoma because of the scarcity of the disease and the variety of clinical and biological characteristics. A multidisciplinary treatment that consists of multidrug chemotherapy and surgery is expected to lead to a good prognosis with few complications. Therefore, a clinical trial for patients with intermediate-risk tumors was designed to establish a standard treatment that reduces complications and achieves good outcomes.

Methods: We planned a prospective phase 2, single-arm study of the efficacy of image-defined risk factors (IDRF)based surgical decision and stepwise treatment intensification for patients with intermediate-risk neuroblastomas. For the localized tumor group, IDRF evaluations will be performed after each three-course chemotherapy, and surgery will be performed when appropriate. For patients with metastatic tumors, a total of five chemotherapy courses will be performed, and primary lesions will be removed when the IDRF becomes negative. The primary endpoint is 3-year progression-free survival rate, and the secondary endpoints include 3-year progression-free survival rates and overall survival rates of the localized group and the metastasis group and the incidence of adverse events. From international results, $75 \%$ is considered an appropriate 3-year progression-free survival rate. If this trial's expected 3-year progression-free survival rate of $85 \%$ is statistically greater than $75 \%$ in the lower limit of the $95.3 \%$ confidence interval, with an accuracy 10\% (85 $\pm 10 \%)$, both groups require more than 65 patients.
\end{abstract}

Discussion: This study is the first clinical trial on the efficacy of IDRF-based surgical decision and stepwise treatment intensification for patients with intermediate-risk neuroblastomas. We expect that this study will contribute to the establishment of a standard treatment for patients with intermediate-risk neuroblastoma.

\footnotetext{
* Correspondence: iehara@koto.kpu-m.ac.jp

'Department of Pediatrics, Graduate School of Medical Science, Kyoto

Prefectural University of Medicine, Kajii-cho, Kawaramachi-Hirokoji, Kamigyo-ku, Kyoto 602-8566, Japan

Full list of author information is available at the end of the article
}

(c) The Author(s). 2020 Open Access This article is licensed under a Creative Commons Attribution 4.0 International License, which permits use, sharing, adaptation, distribution and reproduction in any medium or format, as long as you give appropriate credit to the original author(s) and the source, provide a link to the Creative Commons licence, and indicate if changes were made. The images or other third party material in this article are included in the article's Creative Commons licence, unless indicated otherwise in a credit line to the material. If material is not included in the article's Creative Commons licence and your intended use is not permitted by statutory regulation or exceeds the permitted use, you will need to obtain permission directly from the copyright holder. To view a copy of this licence, visit http://creativecommons.org/licenses/by/4.0/ The Creative Commons Public Domain Dedication waiver (http://creativecommons.org/publicdomain/zero/1.0/) applies to the data made available in this article, unless otherwise stated in a credit line to the data. 
(Continued from previous page)

Trial registration: UMIN000004700, jRCTs051180203; Registered on December 9, 2010.

Keywords: Neuroblastoma, Intermediate risk, IDRF

\section{Background}

Neuroblastoma is the most common pediatric solid tumor, except for brain tumors [1], with about 200 newly diagnosed children each year in Japan. The prognosis of patients with neuroblastoma is strongly related to the age at diagnosis, stage, and biological factors [2-5]. In general, intermediate-risk tumors are mixed groups of localized unresectable tumors at diagnosis without $M Y C N$ gene amplification, and the survival of patients with distant metastases without MYCN gene amplification is less than 12 months. Because the intermediate risk group includes tumors with various clinical and biological characteristics, no clinical trial has been conducted in Japan, and a standard therapy has not been established. Therefore, a clinical trial was designed to establish a standard treatment for patients with intermediate-risk neuroblastoma.

It has been reported that complete resection rates decrease in localized cases with surgical risk factors and surgery-related complications increase [6]. The concept of image-defined risk factors (IDRF) has been proposed internationally as a criterion for estimating the risk of surgery from imaging findings and determining whether removal or biopsy is to be performed as initial surgery [7]. In this clinical trial, one of the goals is to reduce surgical complications while ensuring that the prognosis is not impaired. Therefore, the indication of initial surgery is determined based on IDRF. In addition, evaluations based on IDRF are conducted during delayed primary operations to reduce surgical complications as much as possible. Even if residual tumors are observed at the end of chemotherapy, treatment will be permitted to end if the risk of surgery was considered high according to the IDRF-based assessment. Chemotherapy will be performed for the intermediate risk group as a primary treatment after biopsy.

In the US CCG3881 trial, cisplatin (CDDP), doxorubicin (DXR), cyclophosphamide (CPA), and etoposide (VP-16) were used for patients with localized tumors in the intermediate risk group. According to the results of 228 patients with stage 3 cancer, the survival rate was $100 \%$ for favorable histology, and a $90 \%$ survival rate was reported for patients under 1 year of age with unfavorable histology. In contrast, the survival rate was 54\%, and the prognosis was poor for patients over 1 year of age with unfavorable histologies [8]. Two treatmentrelated deaths were reported during remission.

In the SFOP report in France, chemotherapy with a combination of cyclophosphamide, vincristine, carboplatin, etoposide, and doxorubicin was performed for localized tumors [9]. A trial was conducted in 130 unresectable stage 3 cases, and the 3-year disease-free survival rate was reported to be $89 \%$. Chemotherapy and treatment-related death after surgery have been reported as complications of one case each, and one case of acute renal failure after surgery and one case of Ewing's sarcoma as a secondary cancer developed. Treatment with cyclophosphamide, thiotepa, etoposide, carboplatin, and deferoxamine was reported in the AIEOP of Italy [10]. The study was conducted in 48 unresectable stage 3 cancers in patients over 1 year of age, and the 5-year overall survival rate was $60 \%$. However, this study included 10 patients with $M Y C N$ amplification, of whom seven experienced relapse and died. The treatment results for $M Y C N$ non-amplified cases were not described. Only one case of death was reported for each chemotherapy and surgery.

In the US CCG3881 study, patients with stage 4 cancer who were younger than 1 year of age, without MYCN amplification, showed good results, with a 3year event-free survival (EFS) of 93\% [11]. As complications, four treatment-related deaths were reported, and three patients died from infections. In France, 46 courses of the above treatment were performed for patients with stage 4 cancers who were younger than 1 year of age, and the 5-year EFS of $90 \%$ demonstrates that the treatment was well tolerated and successful in patients without bone metastasis [12]. In this study, among the patients without MYCN amplification and with bone metastasis, high-dose treatment was given to some who did not reach remission by initial treatment, and this report described two chemotherapy-related deaths. On the other hand, POG and CCG in the United States reported on stage 4 patients, at 12 to 18 months of age, respectively. The POG 9047 study treated patients with hyperdiploidy and without MYCN amplifications using CPA, DXR, CDDP, and VP-16 and reported a 4-year EFS of $92 \%$ [13]. One chemotherapy-related death was reported as a treatment complication. In addition, in the CCG 3891 study, treatment with CPA, DXR, CDDP, and VP-16 also resulted in an 86\% 6-year EFS in all patients with stage 4 disease at $12-18$ months of age. The CCG 3891 study assigned high-dose treatment and maintenance as an intensive treatment for patients who were $12-18$ months of age without $M Y C N$ amplifications, but the efficacy of the highdose treatment has not been shown, and there was 
no mention of complications in this report. The above findings demonstrate that, in other countries, the treatment for patients with intermediate-risk tumors consists of cyclic chemotherapy with regimens consisting of two to four agents, but the duration of treatment is inconsistent and the safety is insufficient.

Treatment outcomes for patients with intermediate-risk localized tumors in Japan have not been reported, and the treatment studies for localized tumors that have followed the low-risk infant neuroblastoma protocols 9405 and 9805 have shown only good results [14]. There are no reports on treatment results for infants who are 12-18 months of age with stage 4 tumors without $M Y C N$ amplification in Japan. For infants with stage 4 tumors under 12 months of age, 21 cases have been registered in the 9405 and 9805 studies, and the 4-year EFS was $88 \%$.

To build upon the observations described above, this study aims to investigate an intensified regimen from the infantile neuroblastoma protocols 9405 and 9805 in Japan that used chemotherapy to treat patients with localized tumors. The chemotherapy regimen for patients with metastatic disease was the same as that used in Japanese infant neuroblastoma protocols 9405 and 9805 . While VP-16 is frequently used in other countries, it was not used in this clinical trial due to the associated risk of secondary cancer. We have also found that high-dose chemotherapy with stem cell transplantation should not be given to patients at intermediate risk. However, the following changes are made in consideration of the characteristics of the target case. For the intensity regimen, we have decided to use LI-C consisting of VCR, CPA, and CBDCA for initial treatment for patients with stage 3 cancer, because cases of elder children and of patients of 12-18 months of age with unfavorable histology were also included. However, to prevent confusion, the initial treatment of patients with stage 3 tumors who were 1 year or older will only include the LI-B regimen consisting of VCR, CPA, and THP, which is used in infantile neuroblastoma protocols 9405 and 9805. Furthermore, some treatment responses were improved by using the LI-D regimen that consisted of increasing CPA, THP, and CDDP.

The duration of chemotherapy was 34 weeks in the COG 3881 trial and up to 32 weeks in the new 3961 trial $[11,15]$. Furthermore, the duration of chemotherapies of the France SFOP trial and the Italy AIEOP trial ranged from 12 to 24 weeks, and the treatment periods were generally long $[4,10]$. In this clinical trial, the chemotherapy period was shortened, and treatment was reduced by performing evaluations every three courses. On the other hand, if the treatment response was poor, we will use the LI-E regimen, which had an increased intensity. The treatment periods of this study will range from a minimum of three courses ( 9 weeks) to a maximum of nine courses (34 weeks).

For infants with stage 4 tumors without poor prognostic factors, we used the LI-D regimen of the Japanese infantile neuroblastoma protocol 9405/9805, to reduce treatmentrelated complications. During a total of five to six courses of treatment, distant metastasis is controlled, and surgery is performed when it is possible to remove the primary tumor. Although radiation therapy is not generally used as a treatment for patients at intermediate risk, cases of bone metastases in infants with stage 4 cancer have been reported by SFOP to have a poor prognosis [9]. We therefore plan to irradiate bone metastases for such patients; however, the indications for radiation therapy for the intermediate risk group remain controversial. Although radiation therapy will not be used in this clinical trial, patients with distant bone metastases are permitted to receive irradiation at the discretion of the institution, and this is not considered as protocol withdrawal.

\section{Methods/design Objectives}

This trial aimed to achieve a good prognosis and reduce treatment complications by performing low-dose stepwise chemotherapy and evaluating operation timing, based on IDRF, for patients with intermediate-risk neuroblastoma. This study was conducted according to SPIRIT guidelines.

\section{Study design}

The study is a prospective phase 2 , single-arm study of the efficacy of IDRF-based surgical decision and stepwise treatment intensification for patients with intermediate-risk neuroblastomas. Figure 1 depicts the flow chart of the study. Patients of the localized tumor group will be evaluated by IDRF, and chemotherapy will be given to residual tumors after biopsy. Figure 2 every three courses of chemotherapy, the tumor will be evaluated by IDRF, and surgical removal will be performed when it is determined that there was no risk for surgery. The International Neuroblastoma Response Criteria will be used to determine chemotherapy efficacy. If up to nine courses show MIBG uptake or urinary vanillic mandelic acid (VMA) and homovanillic acid (HVA) do not reach normal values, tumor surgery will be limited to subtotal resection, and treatment will be considered complete. For the metastatic tumor group, a total of five courses of LI$\mathrm{D}$ therapy will be performed and at each evaluation time point. Figure 3 primary tumors will be removed if IDRF is negative, regardless of the tumor marker. However, if it is possible to surgically remove the primary tumor after five therapy courses, a total of six courses of LI-D therapy will be given.

\section{Study setting}

This trial has been approved by ethics review boards at 85 facilities. 


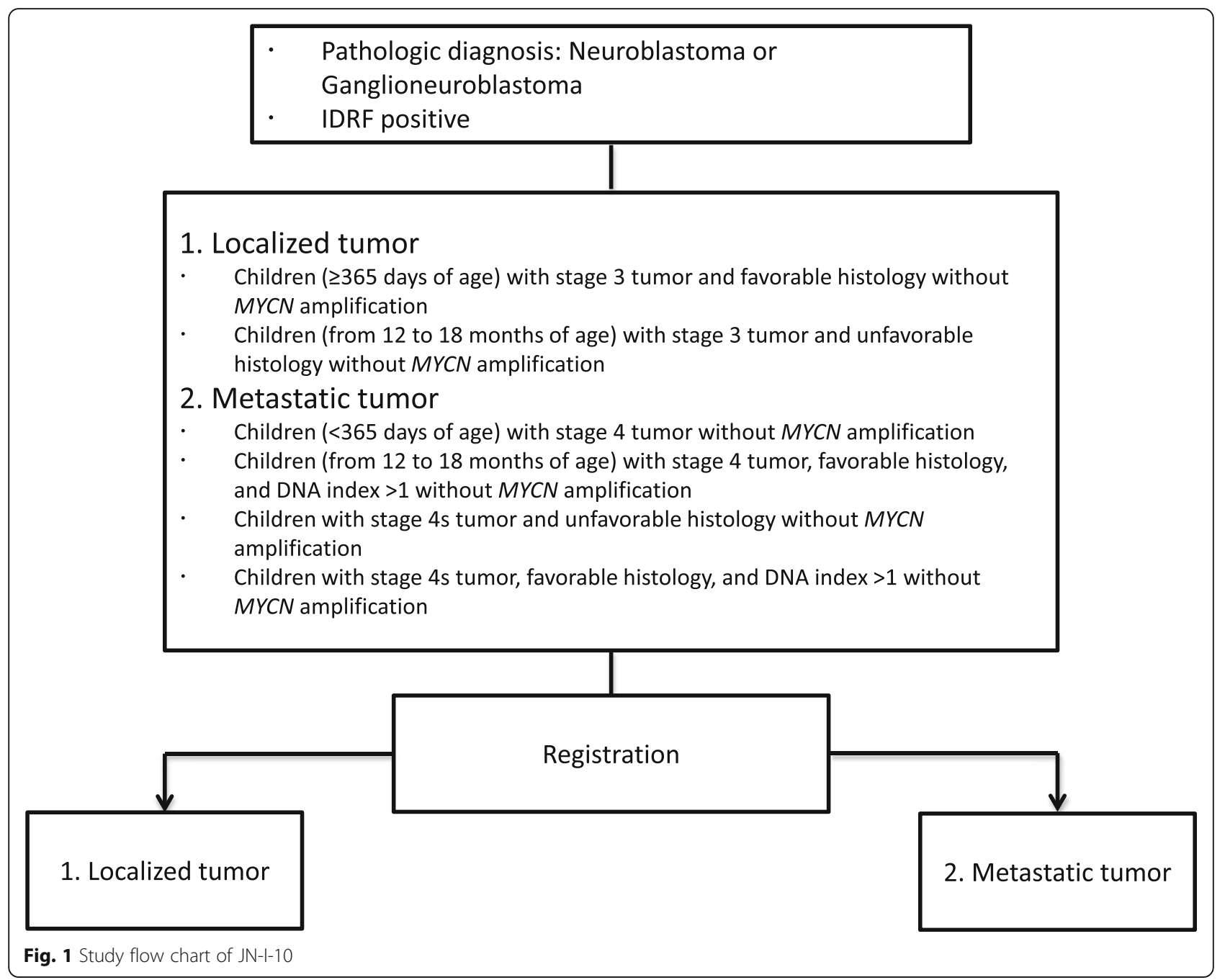

Neuroblastoma is the very rare disease, and about 200 cases with neuroblastoma occur annually in Japan. It is said that only $10 \%$ of cases among them have an intermediate risk neuroblastoma, then the participants must recruit from many centers. The 85 participating facilities consist of 57 university hospitals, 22 Children's hospitals and 6 public hospitals.

\section{Endpoints}

Primary:

Three-year progression-free survival.

Secondary:

We will analyze the 3-year progression-free survival rates of the localized group and of the distant metastasis group. We will also analyze the 3-year overall survival of all eligible patients including those with localized disease, and those with distant metastases. Finally, we will calculate the incidence of adverse events.

\section{Participants}

Inclusion criteria:

(1) Age.

1. $\geq 0$ days, $<18$ years old.

(2) Diagnosis.

- Pathological diagnosis with neuroblastoma or ganglioneuroblastoma.

- Increased urinary catecholamine (VMA, HVA) level and MIBG avid tumor, in cases without tumor biopsy.

(3) Stage, Prognostic factors.

- Stage 3, $\geq 12$ months old, favorable histology, without $M Y C N$ amplification.

- Stage 3, 12-18 months old, unfavorable histology, without MYCN amplification 


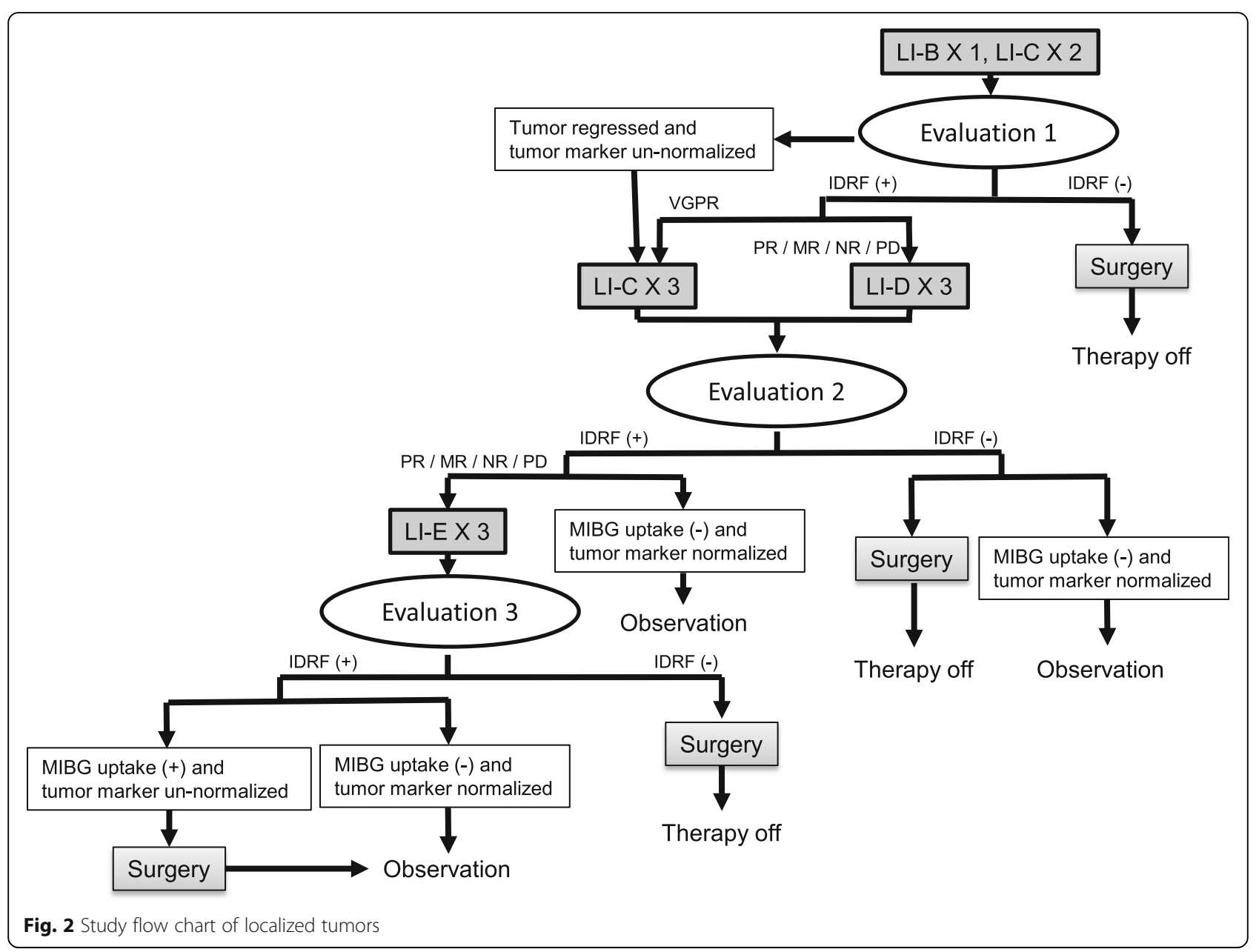

- Stage 4, <12 months old, without $M Y C N$ amplification

- Stage 4, 12-18 months old, favorable histology, DNA index $>1$, without $M Y C N$ amplification

- Stage 4S, unfavorable histology, without $M Y C N$ amplification

- Stage 4S, favorable histology, DNA index =1, without MYCN amplification

(4) Prior treatment.

No history of chemotherapy or radiation, including treatment for other cancers.

(5) Organ failure.

No serious organ damage that interferes with the treatment protocol.

(1) Performance status (PS).

Lansky PS $\geq 30$.

(2) Hematopoietic function.

$\mathrm{WBC} \geq 2000 / \mathrm{mm}$ [3].

(3) Liver function.

$\mathrm{ALT} \leq 300 \mathrm{IU} / \mathrm{L}$ and $\mathrm{T}-\mathrm{Bil} \leq 2.0 \mathrm{mg} / \mathrm{dL}$.
Out of the limitations; neonatal jaundice and jaundice due to this disease; and increase in T-Bil due to constitutional jaundice.

(4) Renal function.

$<5$ years old: serum $\mathrm{Cr} \leq 0.8 \mathrm{mg} / \mathrm{dL}$.

$5-10$ years old: serum $\mathrm{Cr} \leq 1.2 \mathrm{mg} / \mathrm{dL}$.

$10-18$ years old: serum $\mathrm{Cr} \leq 1.5 \mathrm{mg} / \mathrm{dL}$.

(5) Cardiac function.

There are no patients with heart disease who required treatment.

(6) Infection.

No active infections.

(7) Informed consent.

Provision of written informed consent.

\section{Exclusion criteria}

(1) Multiple cancers.

(2) Patients who are pregnant, nursing, or possibly pregnant.

(3) Patients with difficulty participating in the study due to mental illness or mental symptoms. 


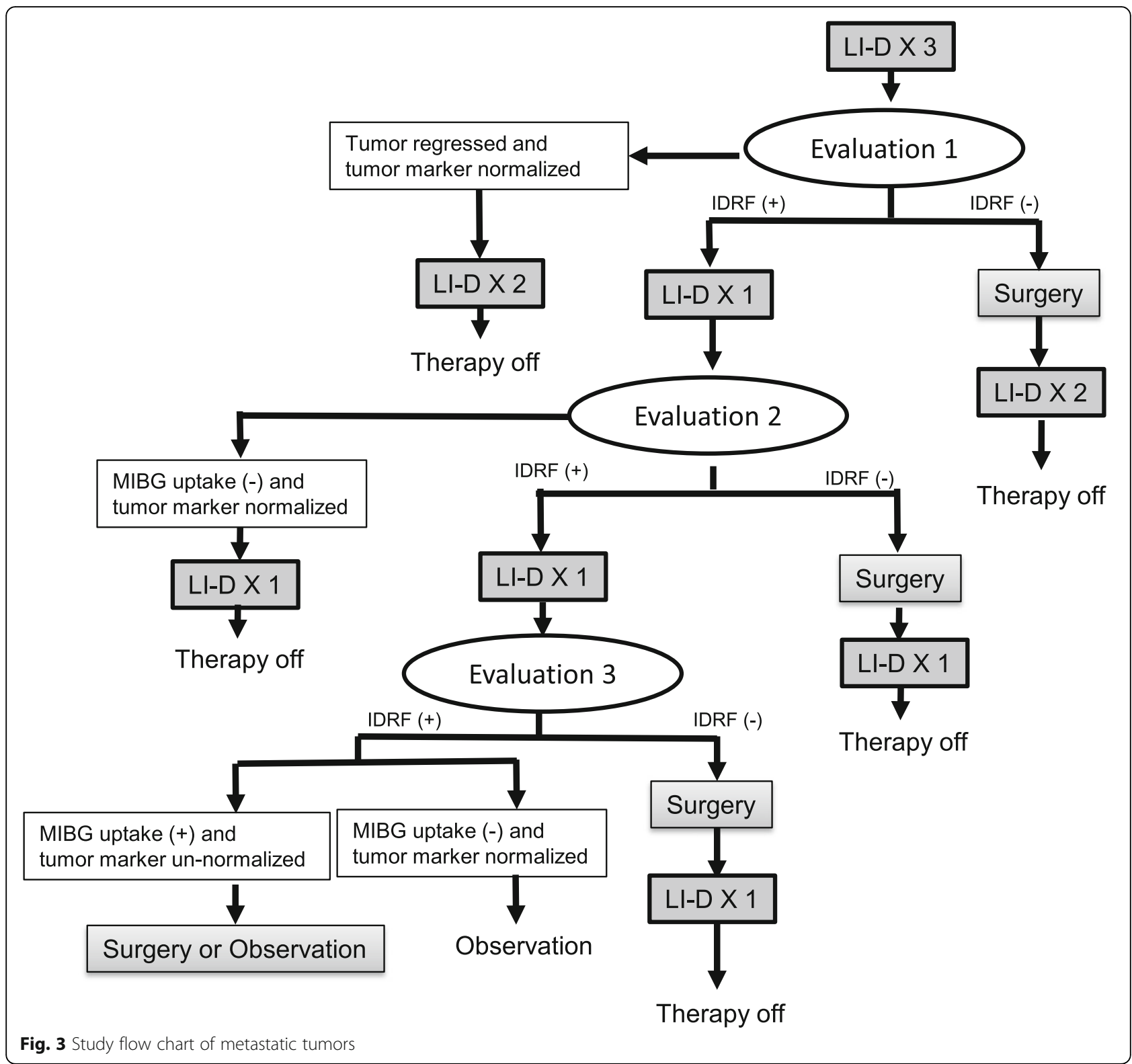

(4) Severe complications or severe malformation.

(5) Respiratory failure requiring respiratory management, such as intubation.

(6) Disseminated Intravascular Coagulation (DIC)

\section{Dose and treatment regimens}

The dose will be calculated based on body surface area as $1 \mathrm{~m}^{2}=30 \mathrm{~kg}$ for patients whose body weight is less than $10 \mathrm{~kg}$.

Regimen LI-B: 3-week interval.

Vincristine (VCR) $1.5 \mathrm{mg} / \mathrm{m}^{2} /$ day day 1 .

Cyclophosphamide (CPA) $600 \mathrm{mg} / \mathrm{m}^{2} /$ day day 1 .

Pirarubicin (THP) $30 \mathrm{mg} / \mathrm{m}^{2} /$ day day 3 .

Regimen LI-C: 3-week interval.

Vincristine (VCR) $1.5 \mathrm{mg} / \mathrm{m}^{2} /$ day day 1 .
Cyclophosphamide (CPA) $900 \mathrm{mg} / \mathrm{m}^{2} /$ day day 1 . Carboplatin (CBDCA) $450 \mathrm{mg} / \mathrm{m}^{2} /$ day day 1 .

Regimen LI-D: 4-week interval. Vincristine (VCR) $1.5 \mathrm{mg} / \mathrm{m}^{2} /$ day day 1 . Cyclophosphamide (CPA) $900 \mathrm{mg} / \mathrm{m}^{2} /$ day day 1 . Pirarubicin (THP) $30 \mathrm{mg} / \mathrm{m}^{2} /$ day day 3 . Cisplatin (CDDP) $12 \mathrm{mg} / \mathrm{m}^{2} /$ day days $1-5$.

Regimen LI-E: 4-week interval. Cyclophosphamide (CPA) $1200 \mathrm{mg} / \mathrm{m}^{2} /$ day day 1 . Pirarubicin (THP) $40 \mathrm{mg} / \mathrm{m}^{2} /$ day day 3 .

Cisplatin (CDDP) $18 \mathrm{mg} / \mathrm{m}^{2} /$ day days $1-5$.

Rationale for the number of enrolled subjects

The 5-year progression-free survival rate for intermediate-risk neuroblastoma in INRG is estimated 
to be 50 to $75 \%$ internationally [16]. On the other hand, for intermediate-risk neuroblastoma (stage 4, < 1 year old) with distant metastasis in Japan, the 3-year progression-free survival rate is 80 to $90 \%$. Therefore, the expected 3-year progression-free survival rate of this study has been set to $85 \%$. Since the international results refer to 5-year progression-free survival rate, the reference 3-year progression-free survival rate has been set to $75 \%$. When the sample size is 65 , a two-sided $95.3 \%$ confidence interval for a single proportion will extend $10 \%$ from the observed proportion for an expected proportion of $85 \%$. The target sample size of this study is 73 , including $10 \%$ patients that will be excluded from the analysis set. Logically, if the treatment outcome is equal to or better than this, this study will be considered to be as effective as a standard treatment.

\section{Statistical methods}

An interim analysis is planned using alpha-spending function after 37 patients (the half of the target sample size) are evaluated. The progression-free survival rates and the confidence intervals are estimated using the Kaplan-Meier method. For adverse event, the worst grade over the entire course of each adverse event in each subject is summarized.

\section{Study population}

All subjects excluding patients with serious violations will be included in this study.

Patients who received anticancer drugs other than those included in the protocol treatment regimen and who received folk remedies for antitumor effects were judged as serious violations.

\section{Regular monitoring}

Regular monitoring is conducted twice a year in order to confirm whether the test is performed safely as planned and in accordance with the protocol, or whether data is collected appropriately. The results of the interim analysis are submitted as an interim analysis report from the data center to the Efficacy and Safety Evaluation Committee, where they are examined for the continuation of the test and the availability of the results.

\section{Trial status}

This study opened recruitment in December 2011, with a planned last follow-up in December 2023. As of December 2020, 73 subjects will be enrolled.

\section{Discussion}

Intermediate-risk neuroblastoma is a rare disease, and few clinical trials have been reported. In addition, since intermediate-risk tumors are heterogeneous and show various tumor dynamics, treatment selection is often difficult. On the other hand, recent reports from the COG group show that the 3-year overall survival of patients with intermediate-risk tumors is as good as $96 \%$, which suggests the possibility for further chemotherapy reduction [15]. We will not use etoposide for chemotherapy or high-dose treatment with stem cell rescue, and we plan to reduce the risk of secondary cancer and complications. With regard to drug doses, patients with localized tumors will receive initial combination therapy with up to three drugs, and we aim to reduce side effects by gradually increasing the intensity. In the induction therapy for high-risk cases in Japan, the 05A3 protocol consisting of CPA, CDDP, VCR, and THP in combination is used, and a high remission rate has been achieved [17]. For metastatic cases, to reduce side effects, we will reduce the CPA and CDDP more than this 05A3 and calculate the dose by weight for infants younger than 1 year of age. We aimed to achieve both good outcomes and reduced side effects.

The tumors of patients in the intermediate risk group include IDRF-positive tumors with surgical risks. Surgery will be delayed as a primary operation after chemotherapy, but few reports from previous clinical studies have described tumor removability and surgical complications in intermediate-risk cases. The operation time decision verifies whether the operation complication can be reduced based on the objective IDRF rather than the subjectivity of the surgeon. The trial design is a nonrandomized phase 2 trial, because it is difficult to increase the number of patients with this rare disease.

The intermediate-risk patients, who were assigned risk classification accordingly, consisted of two subgroups with different characteristics: a localized group and a distant metastasis group. Unfortunately, the number of patients expected in Japan is not large. From previous reports, the number of newly diagnosed patients in Japan has been reported to be 10 patients/year for the localized group and 5.5 patients/year for the metastatic group. Therefore, with approximately 15 patients in both cases, it is difficult to conduct clinical trials. Therefore, in this study, we will explanatively estimate the results of the treatment group for intermediate-risk patients in which the limited and remote groups were combined, and we will select the number of cases based on accuracy. Hence, the number of cases is set such that the 95.3\% confidence interval for the expected 3-year progression-free survival rate of $85 \%$ is $\pm 10 \%$. In this case, the lower limit of the 3-year progression-free survival rate is $75 \%$, which can be compared with the threshold 3-year progression-free survival rate of $75 \%$. Lawless's size design formula requires 65 patients or more and adds $10 \%$ ineligible cases; as such, 73 patients are required. Assuming that the annual expected number of cases is 15, 4.9 years will be required to enroll 15 
patients; therefore, the registration period was originally planned it would be 5 years. The actual registration period may increase or decrease depending on the number of actual ineligible cases. In fact, we found that patients with moderate-risk neuroblastoma were very rare and that recruiting participants could take years, so we revised the registration period to 10 years in 2014.

The results of our clinical trial will allow the establishment of an efficacious and safe standard treatment for patients with intermediate-risk neuroblastoma in Japan.

This study is the first clinical trial of the efficacy of IDRF-based surgical decision and stepwise treatment intensification for patients with intermediate-risk neuroblastomas. We hope that this study will contribute to the establishment of standard treatment for patients with intermediate-risk neuroblastoma.

\section{Abbreviations}

CDDP: Cisplatin; CPA: Cyclophosphamide; DXR: Doxorubicin; EFS: Event-free survival; HVA: Homovanillic acid; IDRF: Image-defined risk factors; JCCG: Japan Children's Cancer Group; PS: Performance status; VMA: Vanillic mandelic acid

\section{Acknowledgements}

The authors gratefully thank the many pediatric oncologists and pediatric surgeons at the participating institutions for enrolling patients. We also thank Editage for the English editing.

\section{Authors' contributions}

$\mathrm{TI}, \mathrm{AY}$ and Ak conceived this study. HT and ST participated in the formal analysis. $\mathrm{HH}$ cpntributed to acquire fundings. TET, TM, KT and SY participated in the investigation. Tl drafted the current manuscript, supervised by TAT. All authors contributed, read and approved the final manuscript.

\section{Funding}

This research was supported in part by the Practical Research for Innovative Cancer Control from the Japan Agency for Medical Research (AMED; \#15ck016130h0002, \#16ck0106130h0003). AMED provided scientific review and funding of this design of the study. AMED provided peer review of this protocol. AMED will not be involved in the collection, analysis, interpretation of data or writing the manuscript.

\section{Availability of data and materials}

Data sharing does not apply to this article as this study is still open for patient enrollment.

However the datasets are available from the corresponding author on reasonable request.

\section{Ethics approval and consent to participate}

The trial has received ethical approval from the Ethics Committee and Clinical Research Review Board of Kyoto Prefectural University of Medicine, Kyoto, Japan (number: C-844, first edition, 21/December/2010, RBMR-C-10581/ 201867, the last edition ver. 2.1, 27/December/2018). This ethical approval covers all the 85 participant institutions. This study is in compliance with the principles of the Declaration of Helsinki and registered in the University Hospital Medical Information Network database (UMIN000004700) (https://upload.umin.ac.jp/cgi-open-bin/ctr_e/index.cgi) and Japan Registry of Clinical trials (jRCTs051180203). Written informed consent is to be obtained from patient and/or a parent or guardian for patient under 16 years old.

\section{Consent for publication}

Not applicable.

\section{Competing interests}

Akihiro Yoneda is a member of the editorial board as an Associate Editor. The other authors declare that they have no competting interests.

\section{Author details}

1Department of Pediatrics, Graduate School of Medical Science, Kyoto Prefectural University of Medicine, Kajii-cho, Kawaramachi-Hirokoji, Kamigyo-ku, Kyoto 602-8566, Japan. ${ }^{2}$ Department of Pediatric Surgery, Osaka City General Hospital, Osaka, Japan. ${ }^{3}$ Department of Pediatric Oncology, Fukushima Medical University Hospital, Fukushima, Japan. ${ }^{4}$ Department of Pediatric Surgery, Kirishima Medical Center, Kagoshima, Japan. ${ }^{5}$ Department of Pediatric Surgery, Kyoto Prefectural University of Medicine, Graduate School of Medical Science, Kyoto, Japan. ${ }^{6}$ National Institute of Public Health, Saitama, Japan. ${ }^{7}$ Department of Biostatistics, Kyoto Prefectural University of Medicine, Graduate School of Medical Science, Kyoto, Japan. ${ }^{8} \mathrm{Clinical}$ Epidemiology Research Center for Pediatric Cancer, National Center for Child Health and Development, Tokyo, Japan.

Received: 1 February 2020 Accepted: 31 March 2020

Published online: 12 May 2020

\section{References}

1. Brodeur GM, Maris JM. Neuroblastoma. In: Pizzo PA, Poplack DG, editors. Principle and practice of pediatric oncology. 4th ed. Philadelphia, PA: Lippincott; 2002. p. 895-937.

2. Adams GA, Shochat SJ, Smith El, Shuster JJ, Joshi W, Altshuler G, et al. Thoracic neuroblastoma: a pediatric oncology group study. J Pediatr Surg. 1993;28:372-7.

3. Evans AE, Albo V, D'Angio GJ, Finklestein JZ, Leiken S, Santulli T, et al. Factors influencing survival of children with nonmetastatic neuroblastoma. Cancer. 1976;38:661-6.

4. Hayes FA, Green A, Hustu HO, Kumar M. Surgicopathologic staging of neuroblastoma: prognostic significance of regional lymph node metastases. J Pediatr. 1983;102:59-62.

5. Cotterill SF, Pearson AD, Pritchard J, Foot AB, Roald B, Kohler JA, et al. Clinical prognostic factors in 1277 patients with neuroblastoma: results of the European neuroblastoma study group 'survey' 1982-1992. Eur J Cancer. 2000;36:901-8.

6. Cecchetto G, Mosseri V, De Bernardi B, Helardot P, Monclair T, Costa E, et al. Surgical risk factors in primary surgery for localized neuroblastoma: the LNESG1 study of the European International Society of Pediatric Oncology neuroblastoma Group. J Clin Oncol. 2005;23:8483-9.

7. Monclair T, Brodeur GM, Ambros PF, Brisse HJ, Cecchetto G, Holmes K, et al. The international neuroblastoma risk group (INRG) staging system: an INRG task force report. J Clin Oncol. 2009;27:298-303.

8. Matthay KK, Perez C, Seeger RC, Brodeur GM, Shimada H, Atkinson JB, et al. Successful treatment of stage III neuroblastoma based on prospective biologic staging: a Children's group study. J Clin Oncol. 1998;16:1256-64.

9. Rubie H, Michon J, Plantaz D, Peyroulet MC, Coze C, Frappaz D, et al. Unresectable localized neuroblastoma: improved survival after primary chemotherapy including carboplatin-etoposide. Br J Cancer. 1998;77:2310-7.

10. Garaventa A, Boni L, Lo Piccolo MS, Tonini GP, Gambini C, Mancini A, et al. Localized unresectable neuroblastoma: results of treatment based on clinical prognostic factors. Ann Oncol. 2002;13:956-64.

11. Schmidt ML, Lukens JN, Seeger RC, Brodeur GM, Shimada H, Gerbing RB, et al. Biologic factors determine prognosis in infants with stage IV neuroblastoma: a prospective Children's Cancer group study. J Clin Oncol. 2000;18:1260-8.

12. Minard V, Hartmann O, Peyroulet MC, Michon J, Coze C, Defachelle AS, et al. Adverse outcome of infants with metastatic neuroblastoma, MYCN amplification and/or bone lesions: results of the French Society of Pediatric Oncology. Br J Cancer. 2000;83:973-9.

13. George RE, London WB, Cohn SL, Maris JM, Kretschmar C, Diller L, et al. Hyperdiploidy plus nonamplified MYCN confers a favorable prognosis in children 12 to 18 months old with disseminated neuroblastoma: a pediatric oncology group study. J Clin Oncol. 2005;23:6466-73.

14. Iehara T, Hamazaki M, Tajiri T, Kawano Y, Kaneko M, Ikeda H, et al. Successful treatment of infants with localized neuroblastoma based on their MYCN status. Int J Clin Oncol. 2013;18:389-95.

15. Baker DL, Schmidt ML, Cohn SL, Maris JM, London WB, Buxton A, et al. Outcome after reduced chemotherapy for intermediate-risk neuroblastoma. N Engl J Med. 2010;363:1313-23.

16. Cohn SL, Pearson AD, London WB, Monclair T, Ambros PF, Brodeur GM, et al. The international neuroblastoma risk group (INRG) classification system: an INRG task force report. J Clin Oncol. 2009;27:289-97. 
17. Hishiki T, Matsumoto K, Ohira M, Kamijo T, Shichino H, Kuroda T, et al. Results of a phase II trial for high-risk neuroblastoma treatment protocol JNH-07: a report from the Japan childhood Cancer group neuroblastoma committee (JNBSG). Int J Clin Oncol. 2018;23:965-73.

\section{Publisher's Note}

Springer Nature remains neutral with regard to jurisdictional claims in published maps and institutional affiliations.

Ready to submit your research? Choose BMC and benefit from:

- fast, convenient online submission

- thorough peer review by experienced researchers in your field

- rapid publication on acceptance

- support for research data, including large and complex data types

- gold Open Access which fosters wider collaboration and increased citations

- maximum visibility for your research: over $100 \mathrm{M}$ website views per year

At BMC, research is always in progress.

Learn more biomedcentral.com/submissions 